\title{
HIGH FIDELITY SIMULATIONS OF UNSTEADY FLOW THROUGH TURBOPUMPS AND FLOWLINERS
}

\author{
(Abstract Submitted to 44th AIAA Aerospace Science Meeting) \\ Cetin C. Kiris, Dochan Kwak, William Chan, and Jeff Housman \\ NASA Advanced Supercomputing (NAS) Division \\ NASA Ames Research Center, Moffett Field, CA
}

\begin{abstract}
High fidelity computations were carried out to analyze the orbiter LH2 feedline flowliner. Computations were performed on the Columbia platform which is a 10,240-processor supercluster consisting of 20 Altix nodes with 512 processor each. Various computational models were used to characterize the ubsteady flow features in the turbopump, including the orbiter Low-Pressure-Fuel-Turbopump (LPFTP) inducer, the orbiter manifold and a test article used to represent the manifold. Unsteady flow originating from the orbiter LPFTP inducer is one of the major contributors to the high frequency cyclic loading that results in high cycle fatigue damage to the gimbal flowliners just upstream of the LPFTP. The flow fields for the orbiter manifold and representative test article are computed and analyzed for similarities and differences. The incompressible Navier-Stokes flow solver INS3D, based on the artificial compressibility method, was used to compute the flow of liquid hydrogen in each test article.
\end{abstract}

\section{Introduction}

Liquid rocket turbopumps operate under severe conditions and at very high rotational speeds. The orbiter Low-Pressure-Fuel-Turbopump (LPFTP) creates transient flow features such as reverse flows, tip clearance effects, secondary flows, vortex shedding, junction flows, and cavitation effects. Flow unsteadiness originated from the inducer is considered to be one of the major contributors to the high frequency cyclic loading that results in cycle fatigue. The reverse flow originated at the tip of inducer blade travels upstream and interacts with the bellows cavity. Off-design operation conditions further increases the likelihood of cavitation. Vapor cavities typically form in the inducer, wherein the operating pressure is close to the vapor pressure of the liquid hydrogen. The inherently unsteady nature of some these cavities may lead to coupling of these phenomena with other transient flow features, such as backflow and turbulent boundary layer.

Various computational models and high-fidelity computations were carried out in order to characterize various aspects of the flowfield near the flowliner. Results including 14 inducer rotations of a straight pipe model with the LPFTP inducer and 5.5 inducer rotations of the LPFTP inducer with the addition of upstream and downstream flowliners including 38 slots, an overhang area between the liners, and the bellows cavity have been reported. The incompressible Navier-Stokes flow solver INS3D, based on the artificial compressibility method, was used to compute the flow of liquid hydrogen in each test article. All computations included tip leakage effects and a pump operating condition of $104.5 \%$ RPL powerlevel. The findings include, a significant time-periodic back-flow generated by the inducer reaching 15 $20 \%$ of the tip relocity and a jet flow of $10-15 \%$ of the inducer tip speed which penetrates into the bellows cavity creating an unsteady recirculation region in the cavity. The reverse flow and unsteady 
recirculation regions create an unsteady interaction between the duct and the bellows cavity resulting in high frequency cycle loading. The back-flow also creates swirl in the bellows cavity on the order of $10 \%$ of the inducer tip velocity.

Additionally, the LPFTP inducer with flowliner geometry is further analyzed and 12 inducer rotations have been completed to better represent the time-periodic nature of the flow field. A parametric study is performed to compare the flowfield of the original and proposed modifications to the upstream and downstream slots. The proposed modifications would entail enlargement by polishing to eliminate cracks. The purpose of this study is to determine if significant changes to the flow field would occur due to the enlarged slots. Furthermore, the flow through the orbiter fuel feedline manifold and the flow through representative test article are computed. The objective of simulating the flow field through the manifold and the test article is to characterize the similarities and differences between the two configurations.

\section{Computational Models}

The computational grid system representing the test article consists of a straight inflow pipe extended upstream a length of four times the diameter of the pipe, the flowliner component, followed by the inducer component. The grid system includes 38 upstream slots, 38 downstream slots, and the overhang area between liners and the bellows cavity. It consists of 264 overlapping grids with 65.9 Million grid points. Details of the grid system are shown in figure 1. The flowliner component consists of an axisymmetric chamber around the external wall of the pipe, and two rows of slots in the streamwise direction. Each slot is a rectangular shaped hole with rounded corners. On the outside wall of the chamber are the bellows which are shaped like 10 periods of a sine wave. The bellows carity is connected to the duct via the overhang area and the slots. Two-dimensional overset grids are first created for the bellows, side walls and the overhang area of the bellows cavity. These are then revolved 360 degrees to form the volume grids. Each slot consists of a body-conforming grid and a warped Cartesian core grid in the middle of the hole. The flowliner component alone contains 212 grids and 41 million points.

In order to speed up and automate the grid generation procedure, a script system has been developed to automatically and rapidly perform the various steps of the grid generation process prior to the use of the flow solver. Special procedures were developed to automatically create grids for each component type. The types included in the script are Blade, Pipe, Ring, Nose, Flowliner, and Strut. The Blade component is one of the most common parts of a liquid rocket subsystem and may contain multiple sections of one or more sets of different blades, (e.g. inducer, impeller, diffuser). The Pipe and Ring components are used to connect different blade components. Pipes can be straight or curved and are bounded by the shroud. Rings can only be straight and are bounded by both the hub and the shroud. The Nose component is a cap that fits at the start or'end of the hub. The flowliner is a highly complex part with bellows, and slots. The Strut component consists of multiple blades connected to brackets at the shroud end and a central hub at the other end. The strut component was not used in the test article. 

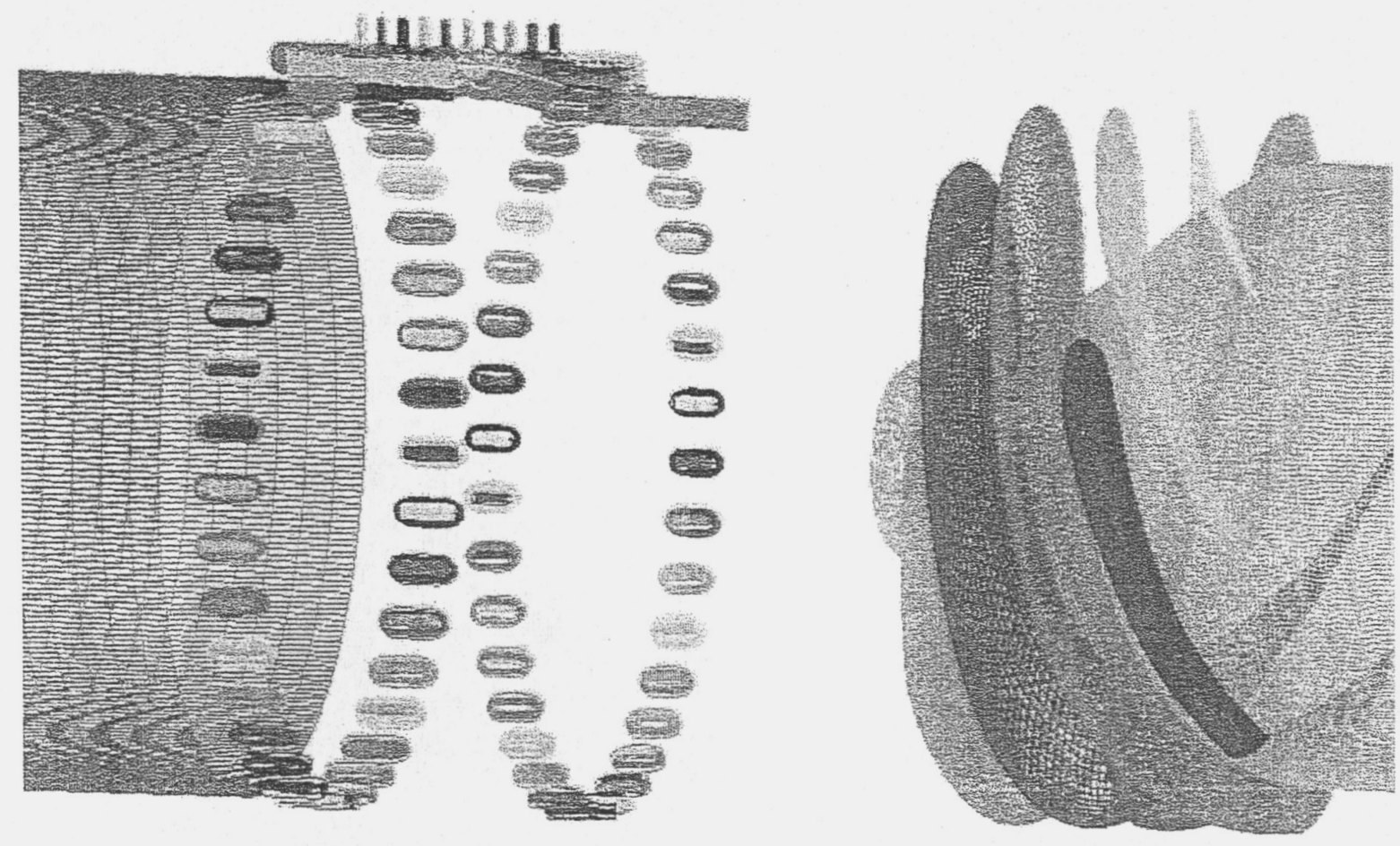

(a)

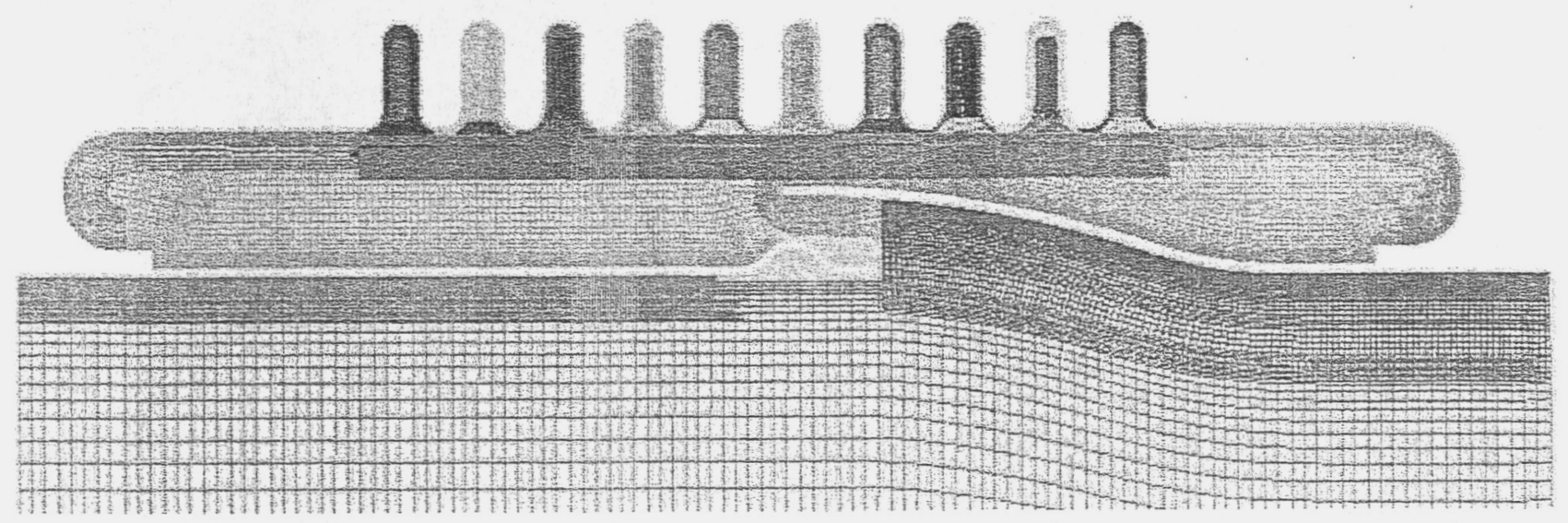

(b)

Figure 1. (a) Surface grids for LPFTP inducer and the Liquid $\mathrm{LH}_{2}$ flowliner. (b) Details of the flowliner overset grid system.

The orbiter fuel feedline manifold grid system consists of an inflow pipe, the manifold, three exit pipes with elbows to the main engines, and two short exit pipes; one for the re-circulating pump and the other for the fill and drain line, (see figure 2(a)). The re-circulating pump and fill and drain line are not included in the computational model so we have closed these two exit pipes. All pipes are connected to the manifold via internal collar grids. The upstream side of the inflow pipe and the downstream side of the exit pipes are modeled by body-conforming $\mathrm{O}$-grids with a singular polar axis running down the core of the pipe. In the regions of the inflow and exit pipes that are near the manifold, the singular axis is avoided by adding a warped Cartesian core grid. Body-conforming grids are used for the walls of the 
manifold. A series of uniform Cartesian grids are used to occupy the core of the manifold. The entire grid system consists of 38 grids and 12 million points, (see figure 2(a) and 2(c)). A separate computational model was generated for the representative manifold test article (see figure 2(b)). The computational grid tepresenting the test article is created using $\mathrm{O}-\mathrm{H}$ grids consisting of six overlapping zones and a total of 7.1 million grid points.

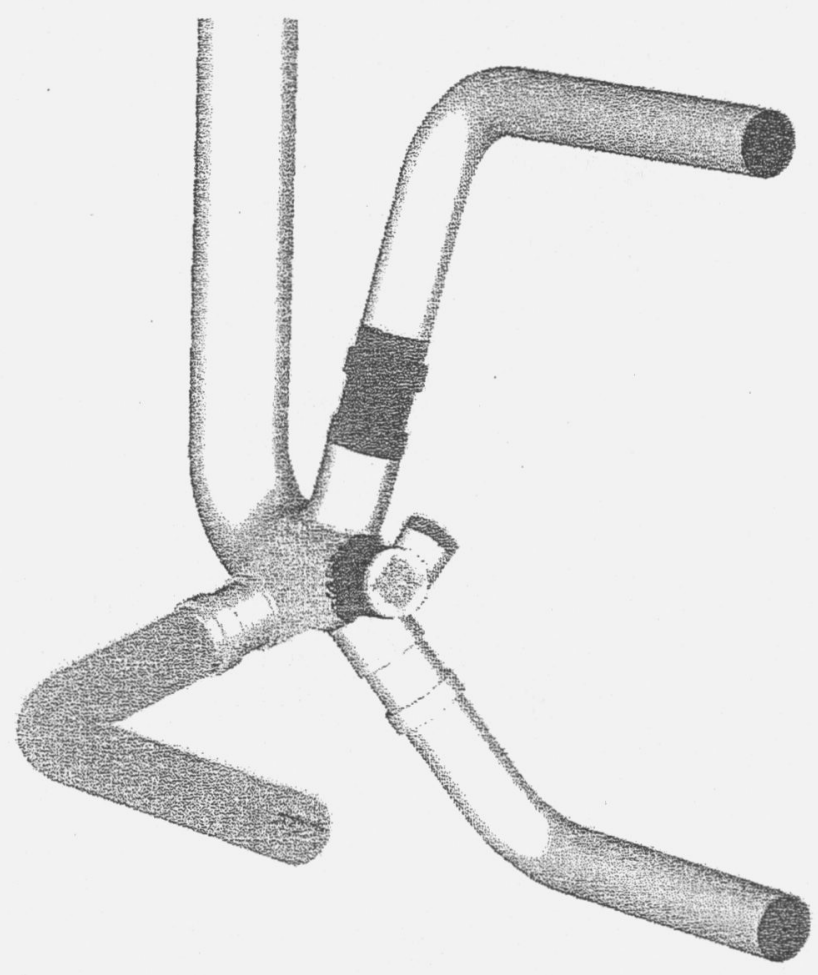

(a)

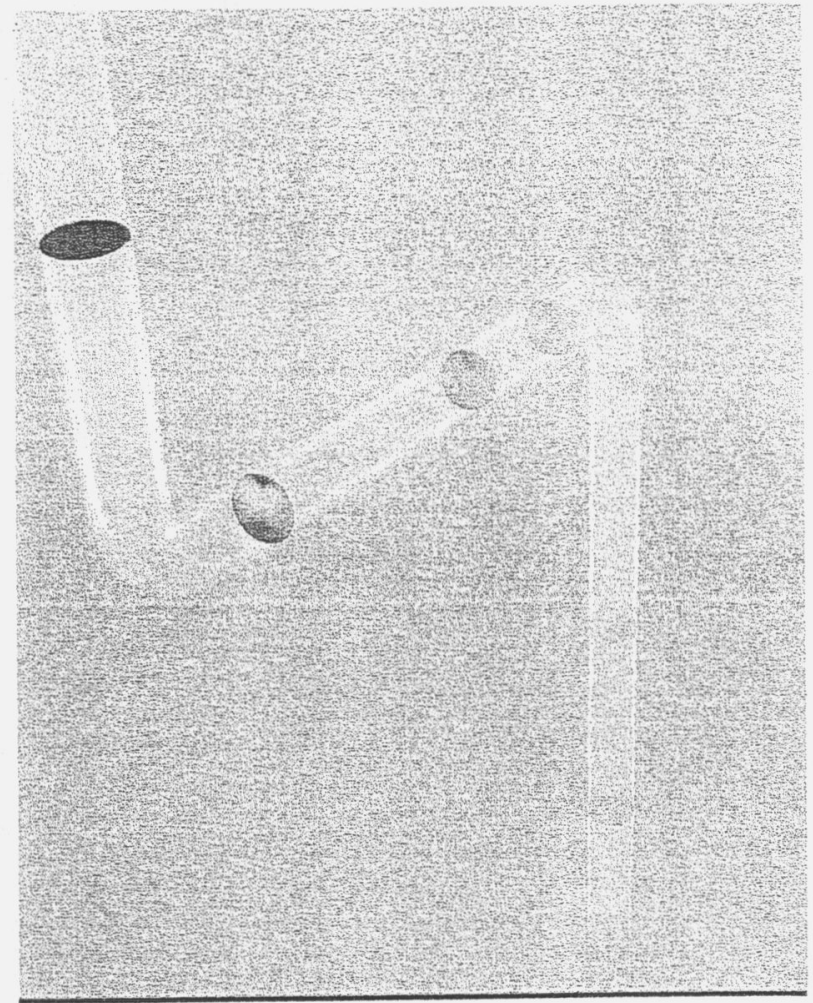

(b)

Figure 2. (a) Details of orbiter fuel feedline manifold, (b) representative test article. 


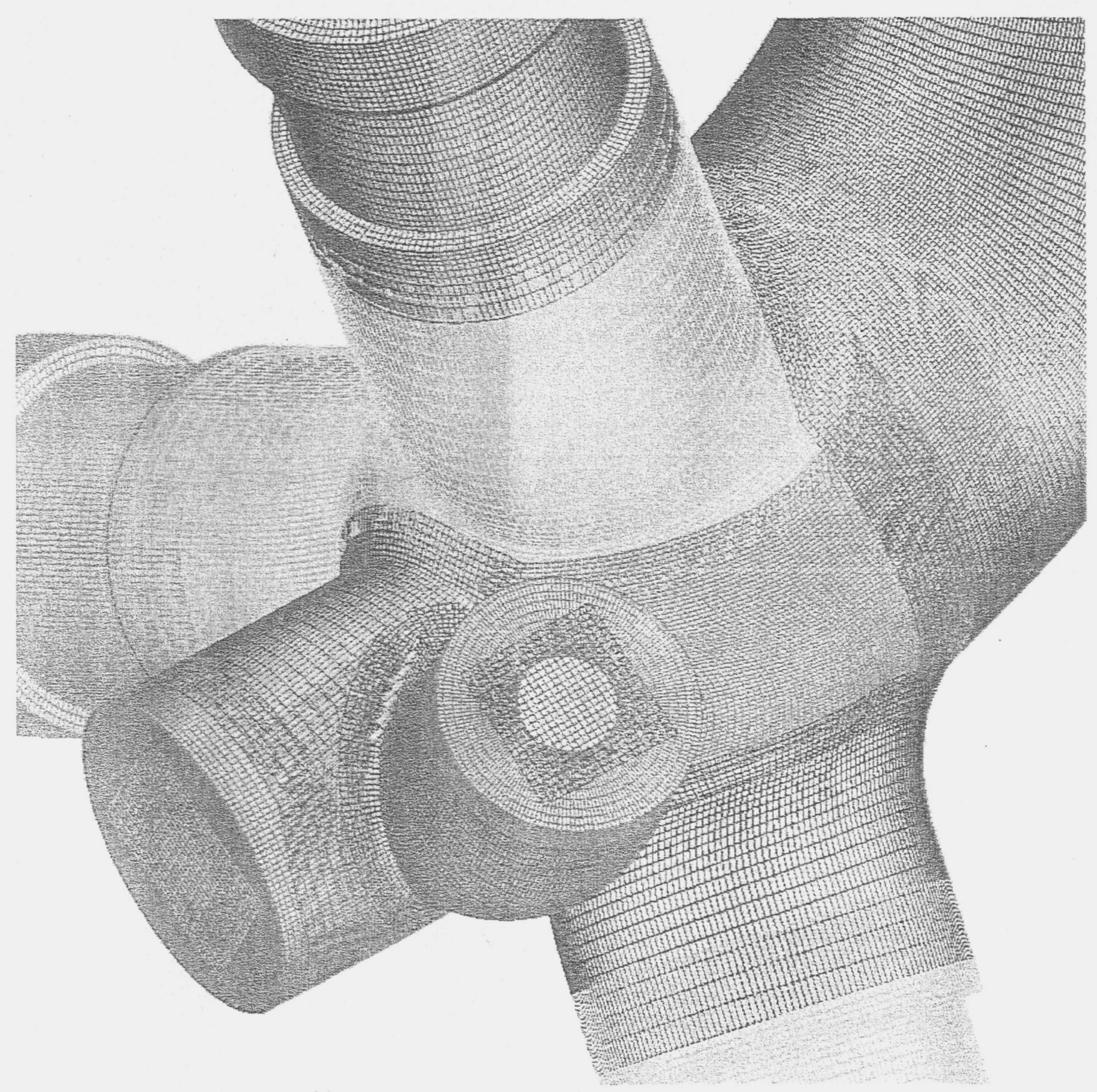

(c)

Figure 2. (c) Details of orbiter fuel feedline manifold.

\section{Numerical Method}

The present computations are performed utilizing the INS3D computer code, which solves the incompressible Navier-Stokes equations for both steady-state and unsteady flows. The numerical solution of the incompressible Navier-Stokes equations requires special attention in order to satisfy the divergence-free constraint on the velocity field. The incompressible formulation does not explicitly yield the pressure field from an equation of state or the continuity equation. One way to avoid the difficulty of 
the elliptic nature of the equations is to use an artificial compressibility method, developed by Chorin ${ }^{3}$. The artificial compressibility method introduces an artificial time-derivative of the pressure into the continuity equation. This transforms the elliptic-parabolic type partial differential equations into the hyperbolic-parabolic type. An incompressible flow solver, INS3D, has been developed ${ }^{2-4}$ based on this algorithm. Since the convective terms of the resulting equations are hyperbolic, upwind differencing can be applied to these terms. The current version uses Roe's flux-difference splitting ${ }^{5}$. The third and fifthorder upwind differencing used here are implementations of a class of high-accuracy flux-differencing schemes for the compressible flow equations. To obtain time-accurate solutions, the equations are iterated to convergence in pseudo-time for each physical time step until the divergence of the velocity field has been reduced below a specified tolerance value. The total number of sub-iterations required varies depending on the problem, time step size and the artificial compressibility parameter used. Typically the number ranges from 10 to 30 sub-iterations. The matrix equation is solved iteratively by using a non-factored Gauss-Seidel type line-relaxation scheme ${ }^{6}$, which maintains stability and allows a large pseudo-time step to be taken. Current computations have been carried out using Multi-Level Parallelism (MIP) on SGI-Origin and SGI-Altix platforms.

\section{Computed Results}

\section{Flowliner Analysis}

In all computations, the inducer tip leakage effect is included and the pump operating condition is 104.5\% RPL power-level. The problem was non-dimensionalized with a reference length of one inch and inducer tip velocity. The Reynolds number for these calculations is 36 Million. Liquid Hydrogen is treated as an incompressible single phase fluid. An initial condition of flow at rest and no inducer rotation is used to start the computations. The inducer is then rotated at full speed as a start-up procedure. Mass flow is specified at the inflow and pressure is extrapolated at the outflow along with a mass-flow correction. Simulations for 12 inducer rotations were completed, we present instantaneous snapshots of particle traces and surface contours of axial and cross-flow velocities in a 3D perspective.

In figure 3(a) particles are released from one upstream slot, one downstream slot, and from the overhang area. In figures $3(\mathrm{~b})-3(\mathrm{~d})$ the particles are released from each of the 38 downstream slots. The mean flow direction is from left to right in the figure 3(a) and figure 3(c), towards the inducer. In figure 3(b) and figure $3(d)$ we are viewing the flowliner from the inducer. The figures are snapshots from particle trace animations that have evolved for one and a half rotations. The particles are colored by the axial velocity values ranging from $-20 \%$ to $+20 \%$ of the inducer tip speed, see (figure $3(\mathrm{a})$ ). The particles released from both upstream and downstream slots in figure 3(a) are interacting with neighboring slots traveling from the duct to the bellows cavity and from the bellows cavity to the duct. This indicates a highly unsteady interaction between the flow field in the bellows cavity and the flow field in the duct. The swirl present in the particle trace appears to be higher in the downstream slots where particles have traced four and half slots while the upstream particles have traveled only two and half slots. The particles released from the overhang area tend to travel into the bellows cavity with the exception of a few particles traveling into the duct. In figure $3(\mathrm{~b})$ we are observing the particles from the inducers perspective where we can see the particles moving into and out of the bellows cavity through the slots. There are four regions of positive velocity particles traveling from the walls toward the center. These are particles released from the slots which do not interact with the four reverse flow regions created by the inducer. Analyzing the interior of the duct we can see the existence of the swirl near the downstream flowliner. 
Examining figure 3(c) we can see that some of the particles released from the slots travel to the overhang region, where they interact with the reverse flow and some of the particles become trapped in the bellows cavity. This suggests that some particles are recirculating through the overhang area into the bellows cavity and through the slots into the duct. This unsteady interaction between four backflow regions generated by the inducer and the secondary flow features in the bellows cavity lead to a break up of the 4 theta forcing function in the flowliner area. Figure 3(d) suggests the existence of a 5 theta forcing function, but more post-processing and future investigations should be conducted.

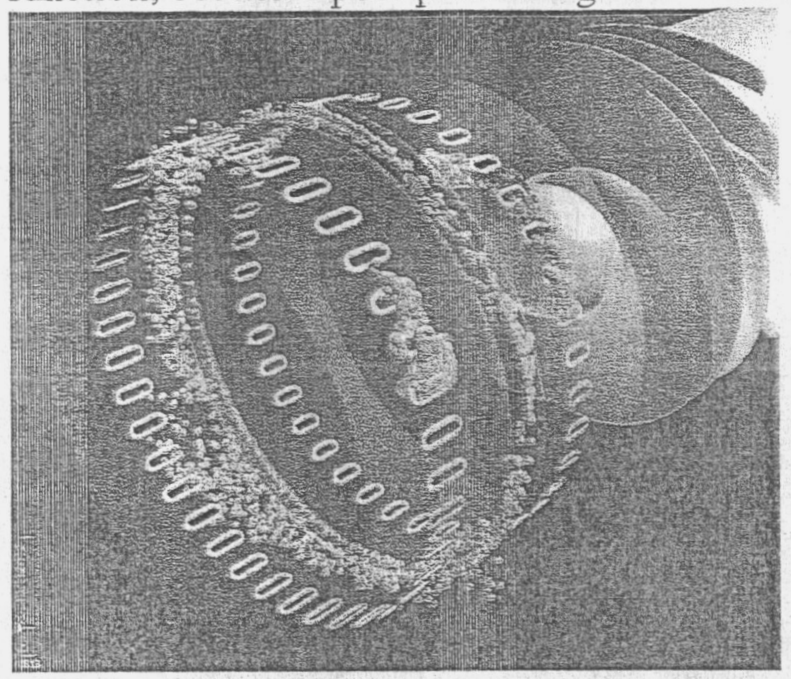

(a)

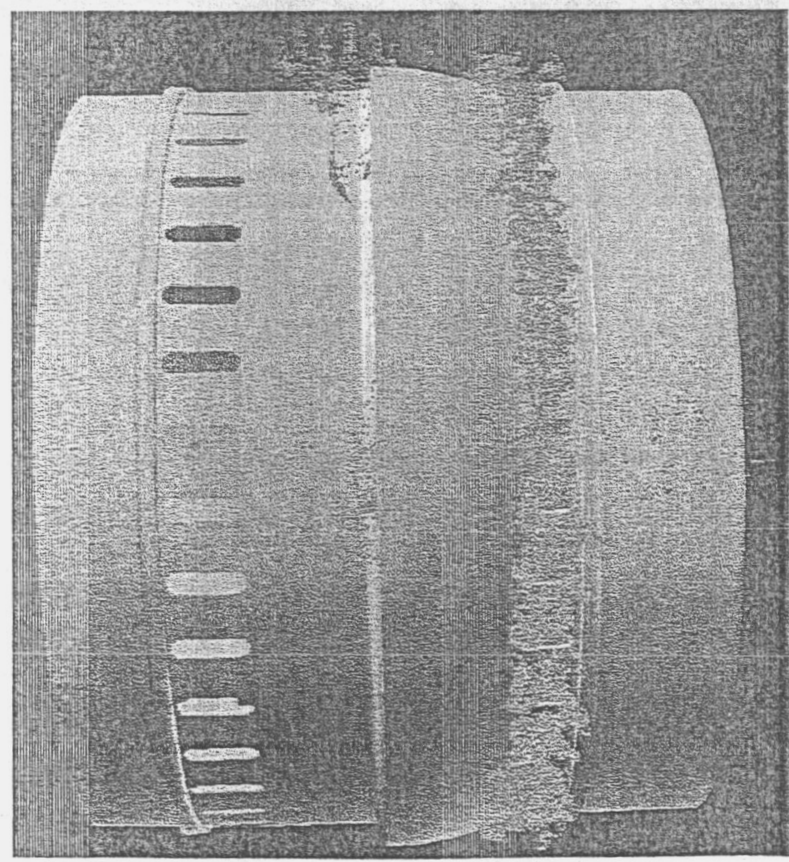

(c)

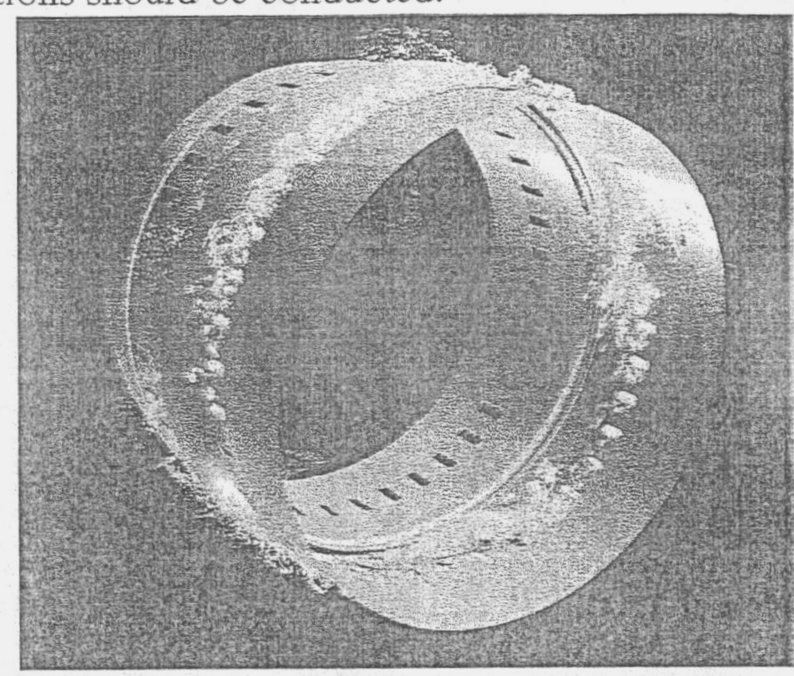

(b)

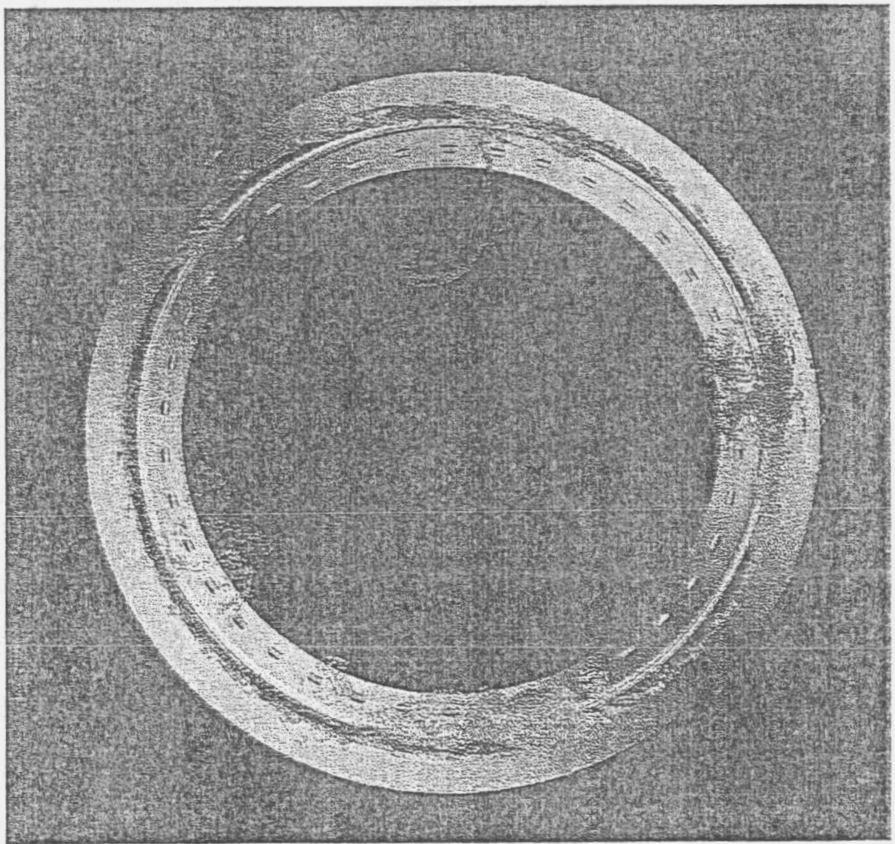

(d)

Figure 3. Instantaneous snapshots of particle traces colored by axial velocity values.

In figures $4(a)$ and $4(b)$ particles are released from the upstream slots and evolve for five inducer rotations. The color of the particles represent forward flow (blue) and backward flow (red). We see from 
the figures that the particles are driven towards the center of the duct and travel to the inducer where some of the particles are trapped into the backflow regions with the 4theta forcing function. In afigures $4(c)$ and $4(d)$ the particles are released from the downstream slots and evolve for five rotations. In figure 4 (c) the particles are colored the same as in the previous two figures. We observe a much more complicated flow structure where many of the particles travel into and out of the bellows cavity. Figure 4(d) we have multi-colored the particles with axial velocity in order to illustrate their presence in the bellows cavity. When the particles are released from the downstream slots they are under the influence of the backflow and swirl such that fewer particles travel towards the inducer.

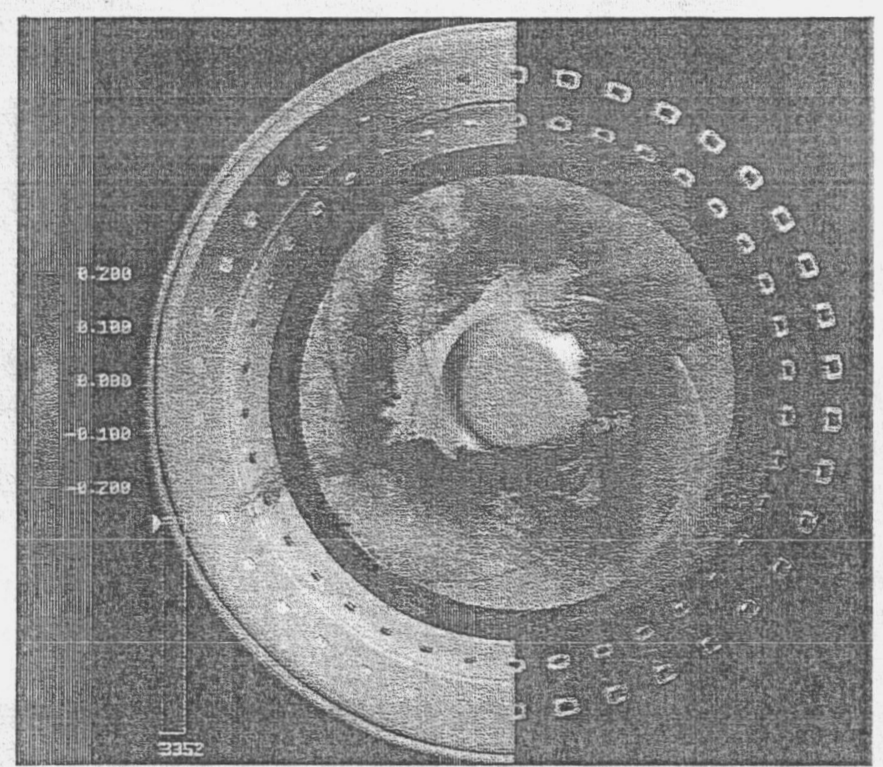

(a)

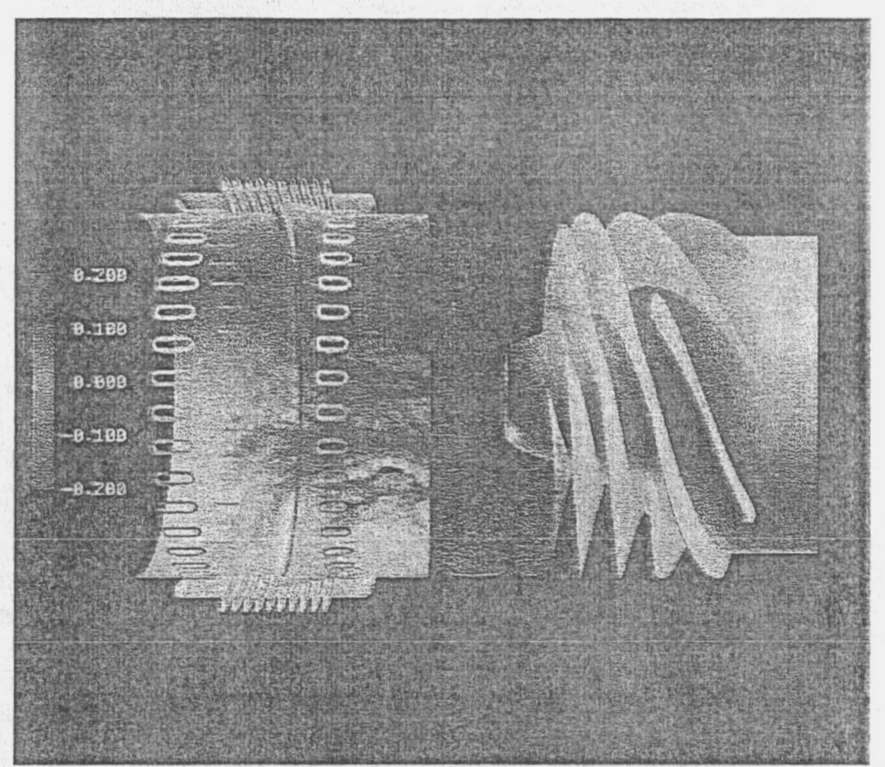

(b)

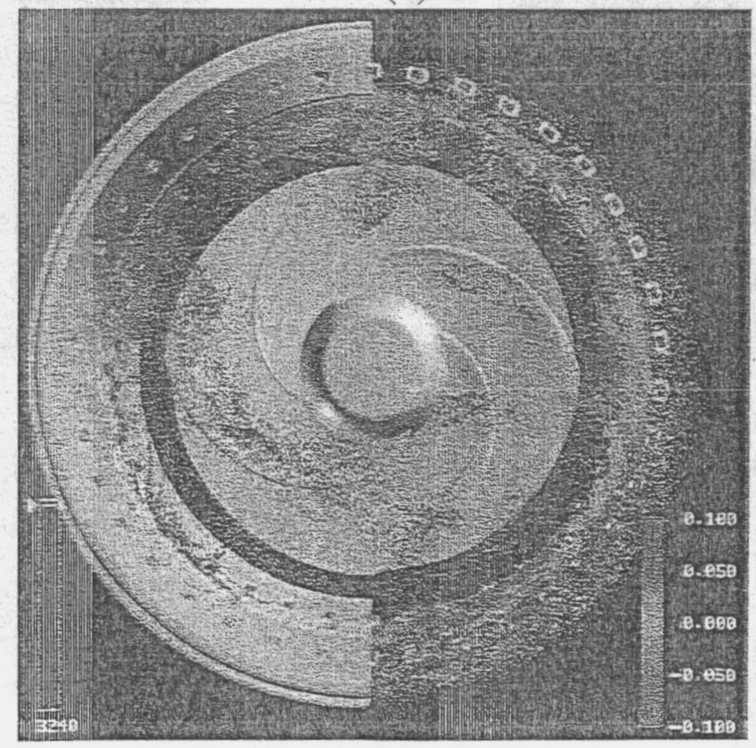

(c)

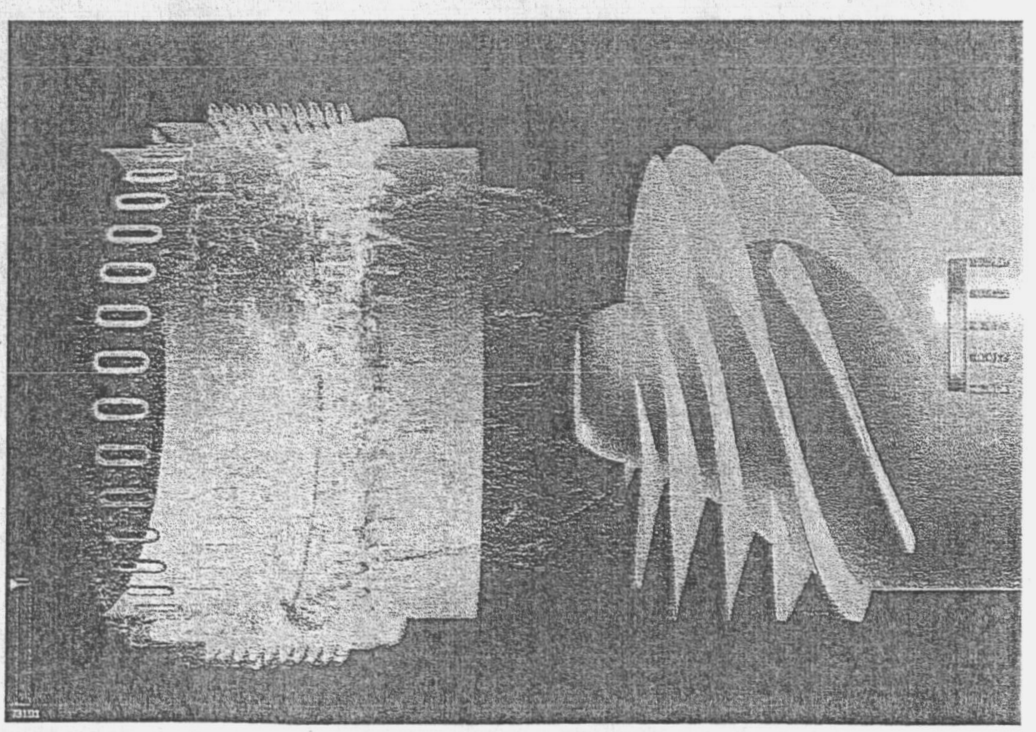

(d)

Figure 4. Instantaneous snapshots of particle traces colored by axial velocity values.

Figure 5 displays the axial velocity contours on a vertical plane. Due to the number of slots, which is 38 instead of 36 , there is a lack of symmetry on the vertical plane. To create this figure the data on each of the structured overset grids are cut vertically and projected onto an unstructured two-dimensional surface. 
Inherent in this process is the creation of small discontinuities in the contours between overset grids that do not line up with one to one matching in the selected two-dimensional plane. The contours show strong reverse flow regions coming from the blade, traveling through the overhang region creating a jet like flow on the order of $10 \%$ of the inducer tip speed. The backflow regions travel up from the inducer blades to the upstream flowliner.

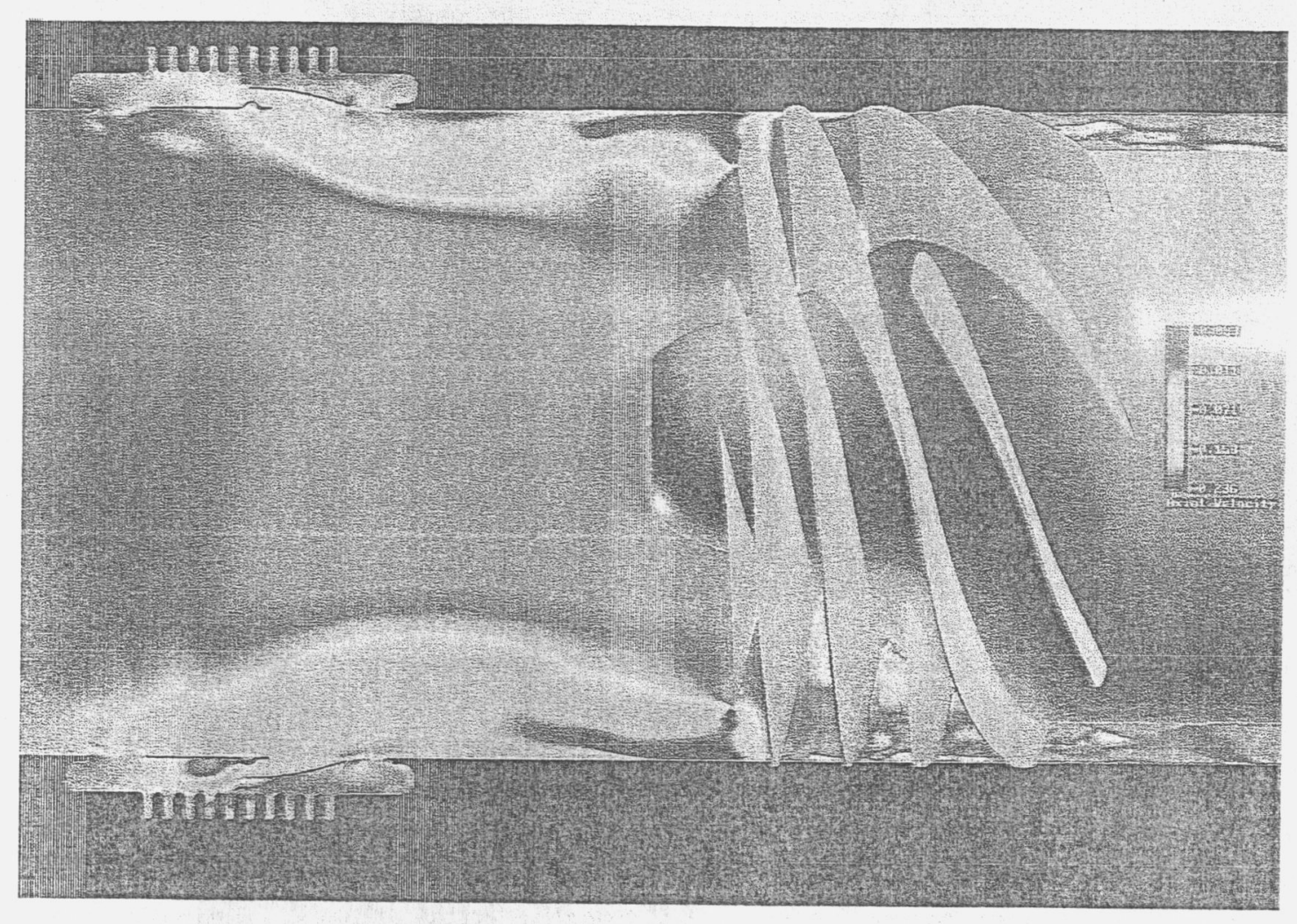

Figure 5. Instaneous axial velocity contours on a vertical plane ( $12^{\text {th }}$ rotation). 


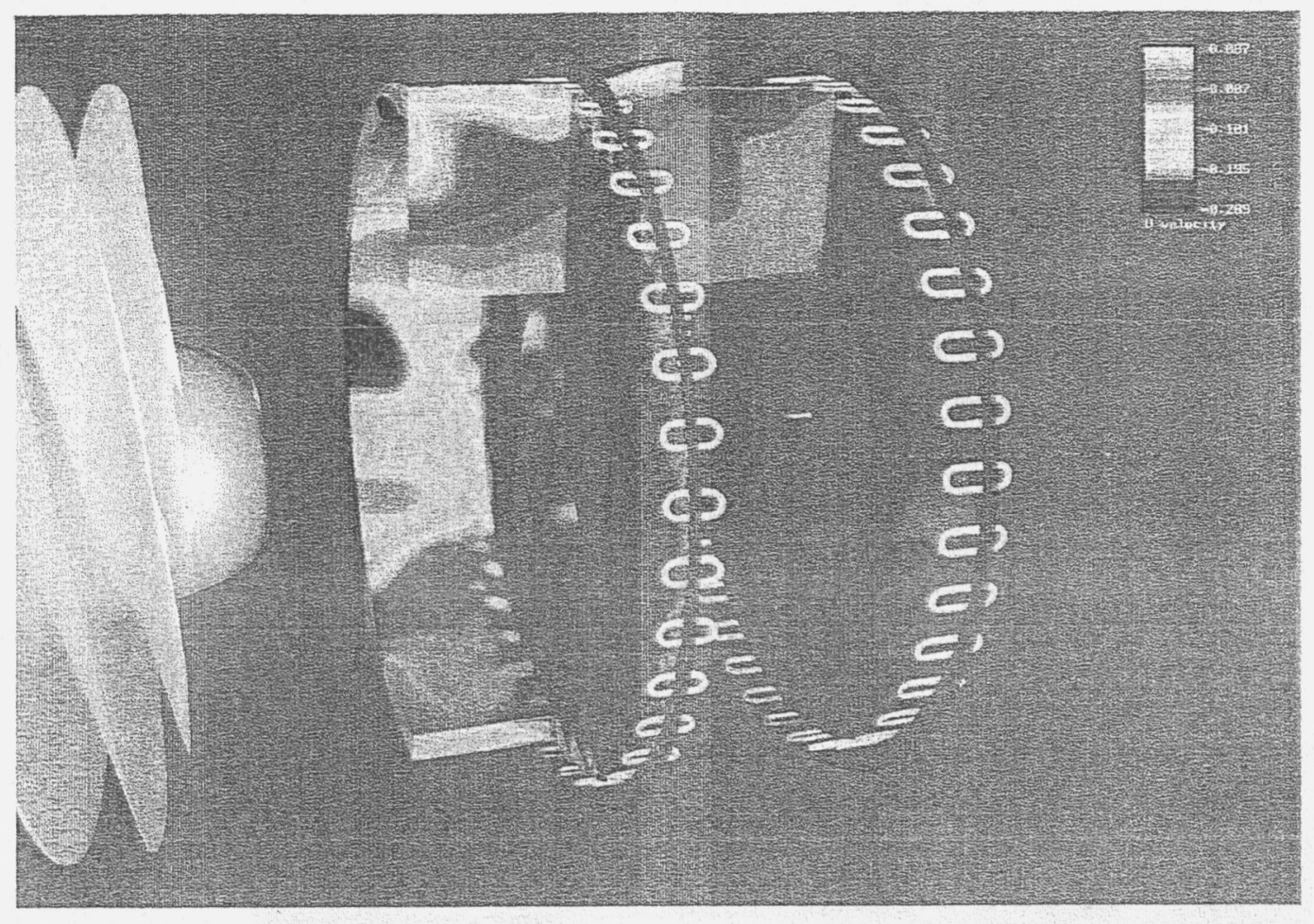

Figure 6. Instantaneous axial velocity contours on vertical, circumferential, and cross-sectional planes.

Figure 6 provides a 3D perspective of the axial flow contours displaying vertical, circumferential, and cross-sectional contour planes. Evidence of a strong reverse flow generated by the blades that travels to the upstream flowliner is seen as in the previous figure. Examining the cross-sectional contour planes surrounding the upstream and downstream flowliners we see stronger reverse flow regions in the downstream flowliner than in the upstream flowliner. Additionally, figure 7 displays the cross flow velocity magnitude at the same cutting planes as the previous figure. The cross flow velocity is highest near the blades of the inducer and coincides with the largest reverse flow regions. At each of the slots of the downstream flowliner high magnitude cross flow velocity contours are found in the bellows cavity and in the duct which provides evidence of the strong unsteady interaction of the flow through the slots between the bellows cavity and the duct. The order of magnitude of the swirl in the duct near the downstream liner is $15 \%$ of the inducer tip speed while at the upstream liner the swirl in the duct is less than $3 \%$ of the inducer tip speed. Alternatively the swirl maintains a higher value in the bellows cavity near the upstream flowliner. This indicates that the swirl travels through the overhang area into the bellows cavity. 


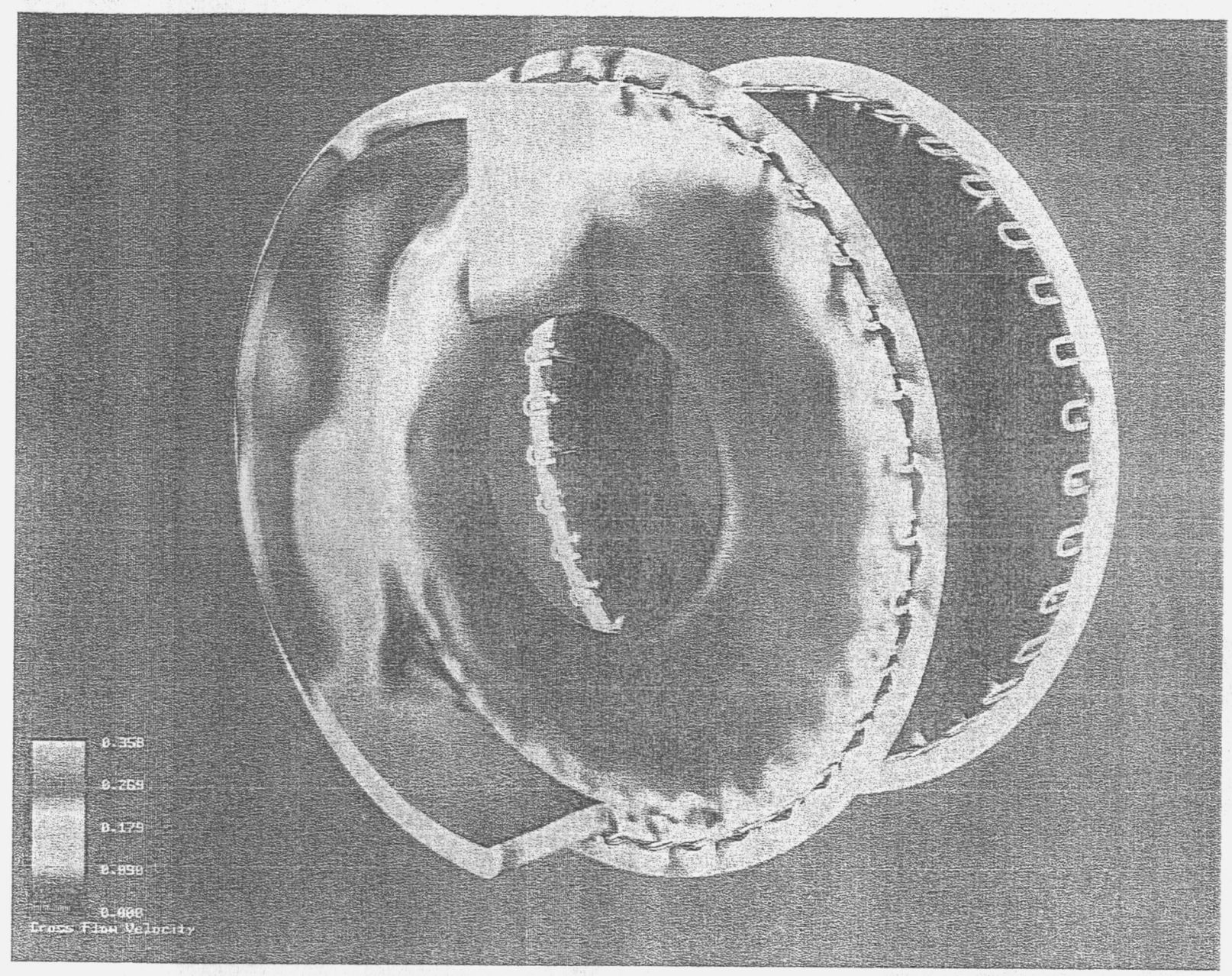

Figure 7. Cross-Flow velocity magnitude at instantaneous time ( $12^{\text {th }}$ rotation).

One of the recommendations to repair any new cracks found on the flowliners is to polish them such that the cracks are removed. This procedure makes the polished slots larger than the original size. A computational model is created such that one upstream flowliner slot and one downstream flowliner slot is $20 \%$ larger in width and $7 \%$ larger in length than the neighboring slots. Initial conditions for this solution were started from the eighth inducer rotation of the original configuration. The solution is then computed for four additional inducer rotations and the solutions for both the original and modified configurations are compared. The purpose of the two computations is to determine if the existence of larger slots than the others creates any significant differences in the flow field. 

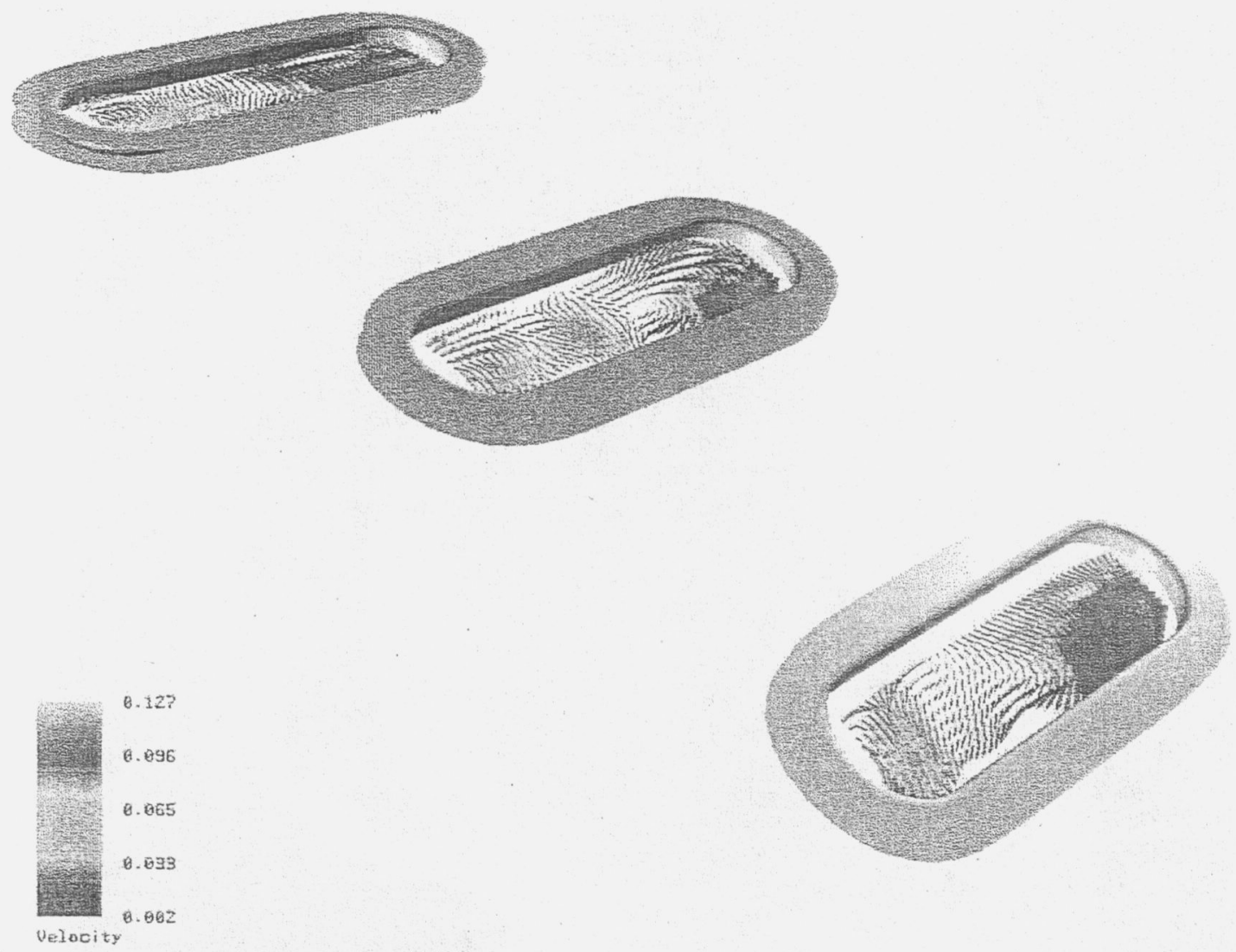

Figure 8. Velocity vectors at instantaneous time in the upstream liner slots (original configuration).
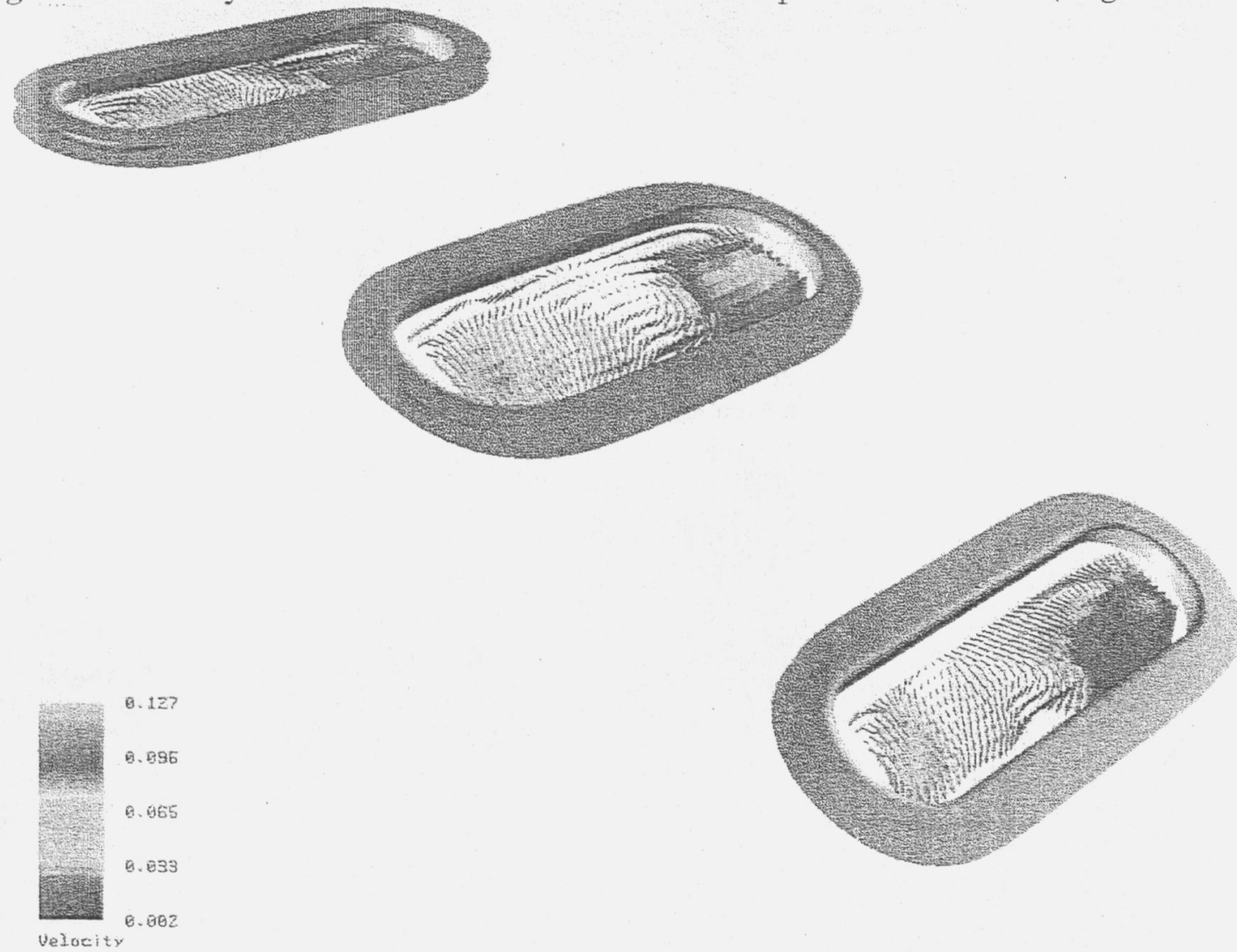

Figure 9. Velocity vectors at instantaneous time in the upstream liner slots (one enlarged slot). 


$$
{ }_{0}^{\circ}
$$


A detailed look at the velocity fields in the slots reveals some quantitative differences. In figure 8 the velocity vectors inside the original upstream liner slots are shown. Analogously, in figure 9 the velocity vectors inside the enlarged upstream slots are displayed. The larger slot is located at the center of the picture. In both designs a large velocity magnitude exists at the upstream edge of each of the slots. In the enlarged slot a slightly higher velocity magnitude region is created near the downstream edge of the slot. Qualitatively, the velocity fields are very similar and it appears that enlarging one slot does not affect the flow field in a significant way. Figures 10 and 11 show the velocity vectors at the original and enlarged downstream flowliner slots at another instantaneous time. The only noticeable difference is the appearance of a secondary flow region near the base of the enlarged slot.

\section{Oribter Manifold and Test Article Analysis}

Initially, steady-state calculations were conducted for both the orbiter manifold and the representative test article. The calculations for the orbiter manifold did not converge to a steady solution because of high grid resolution which captures the fine scale unsteady details that exist in high Reynolds number flows. Then, time-accurate calculations were performed and the mean flow results are presented here. For both of these computations the same non-dimensionalization is used as in the flowliner analysis including a Reynolds number of 36 million based on the inducer tip speed and the reference length of an inch. Consistent inflow and exit boundary conditions are used for the test article and the orbiter manifold, such that the test article exit and the three engines downstream of the orbiter manifold receive the same massflow rate. At the inflow, the mass-flow rate is specified with a corresponding turbulent velocity profile and the pressure is calculated through the characteristic relation. At the exit the pressure is extrapolated and the mass-flow rate is enforced.

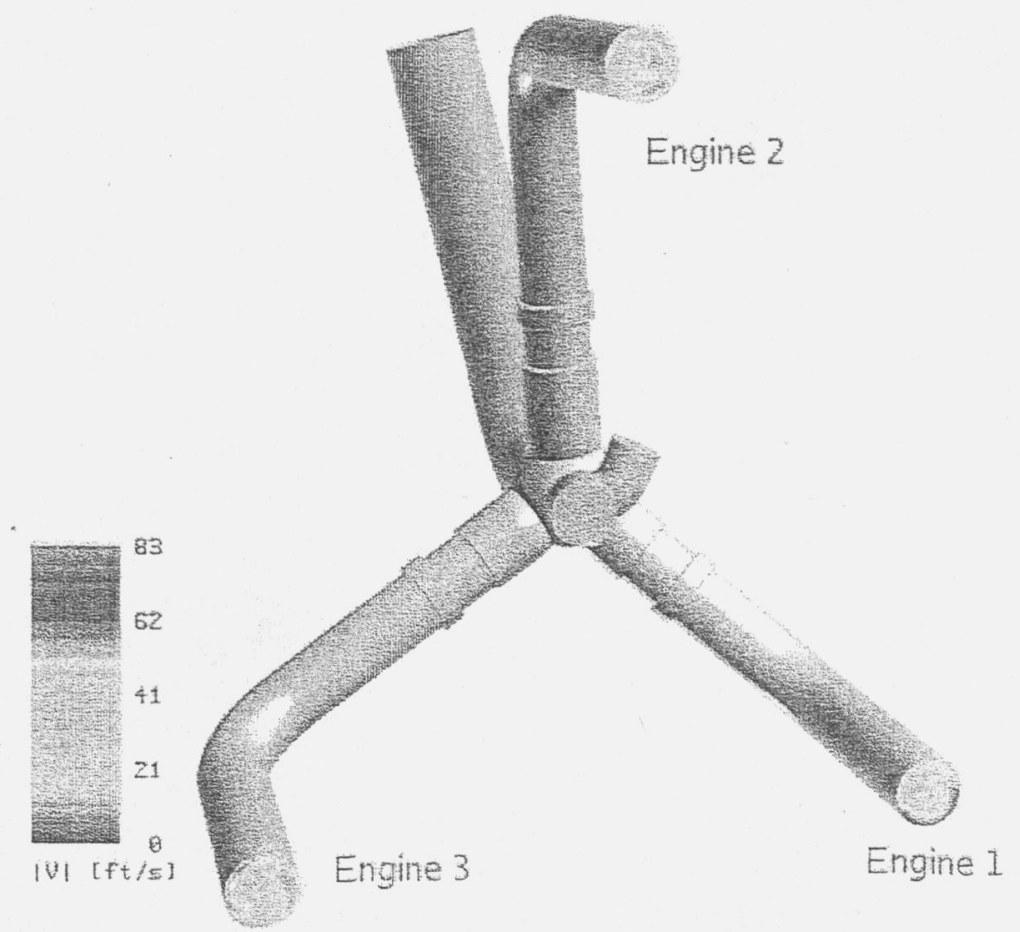

Figure 12. Total velocity magnitude contours at the outlet of the orbiter manifold and inlet to the LPFTP. 


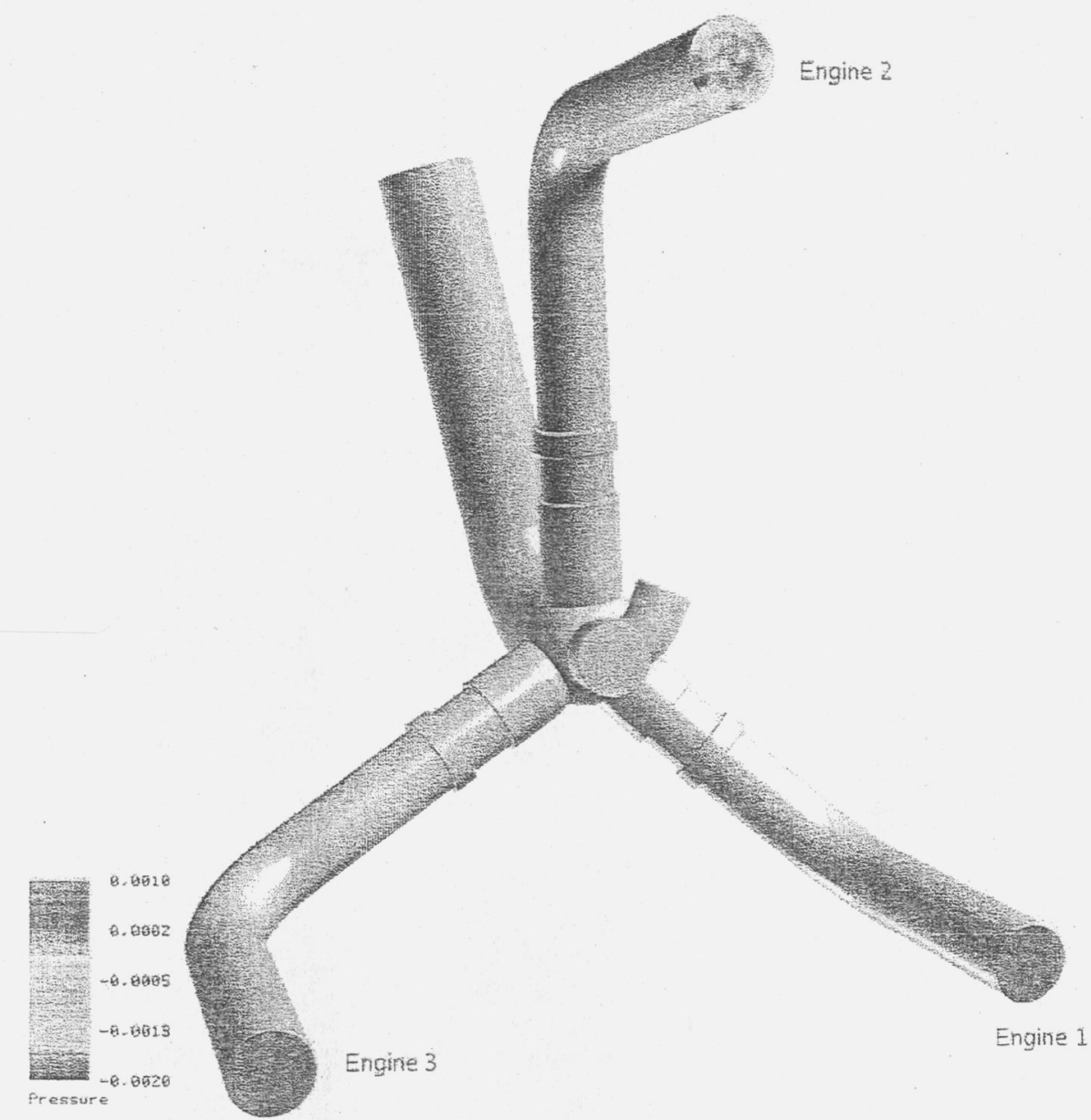

Figure 13. Delta pressure contours at the outlet of the orbiter manifold and inlet to the LPFTP.

The orbiter manifold displayed in figure 12 is qualitatively different for each of the three outlet sections, and none of these sections are represented well by the test article results. Figure 12 also shows that a large velocity profile has developed at the outer wall of the engine 1 feedline, while the high velocity region is better distributed around the entire wall of the engine 3 feedline, and high-low velocity regions near the walls of the engine 2 feedline are not well-developed. The exit pressure in engine 2 has converged to 1.3 psi less than the exit pressure value of engine 1 and engine 3 , see figure 13 . One of the primary reasons for the different velocity profiles and pressure contours at the three engine feedlines is the difference in lengths of the engine feedlines. Additionally the velocity profiles for each of the three engines are different as they leave the manifold.

Figure 14 displays the velocity magnitude on the vertical plane of the test article. A well-developed straight pipe turbulent profile is observed in the inlet and outlet sections. The image shows a very high to very low velocity magnitude on the inside of the first bend, while at the second bend the velocity is more uniform. Figure 15 displays the pressure profile on the vertical plane which looks similar to the pressure profile of an inviscid pipe flow, this is expected from the high Reynolds number. The vertically sliced velocity and pressure profiles obtained from the orbiter manifold solution are qualitatively different then those computed in the test article.

The velocity magnitude contours shown in figure 16 and pressure contours shown in figure 17 illustrates that each engine LPFTP receives a different inflow velocity profile and a different inlet pressure than observed for the test article. 

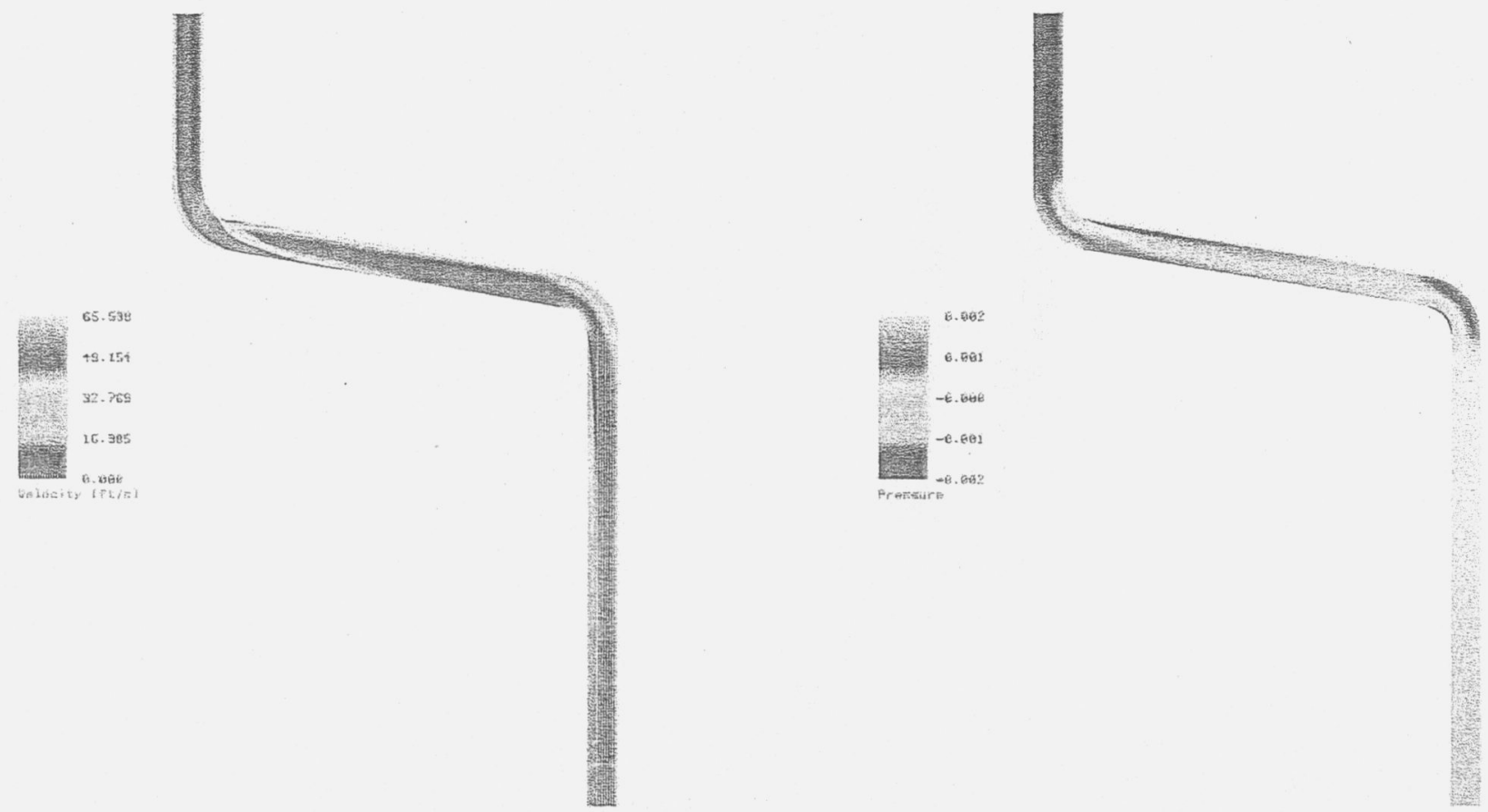

Figüre 14. Total velocity magnitude contours on the vertical plane of the AI test stand.

Figure 15. Pressure contours on the vertical plane of the Al test stand.

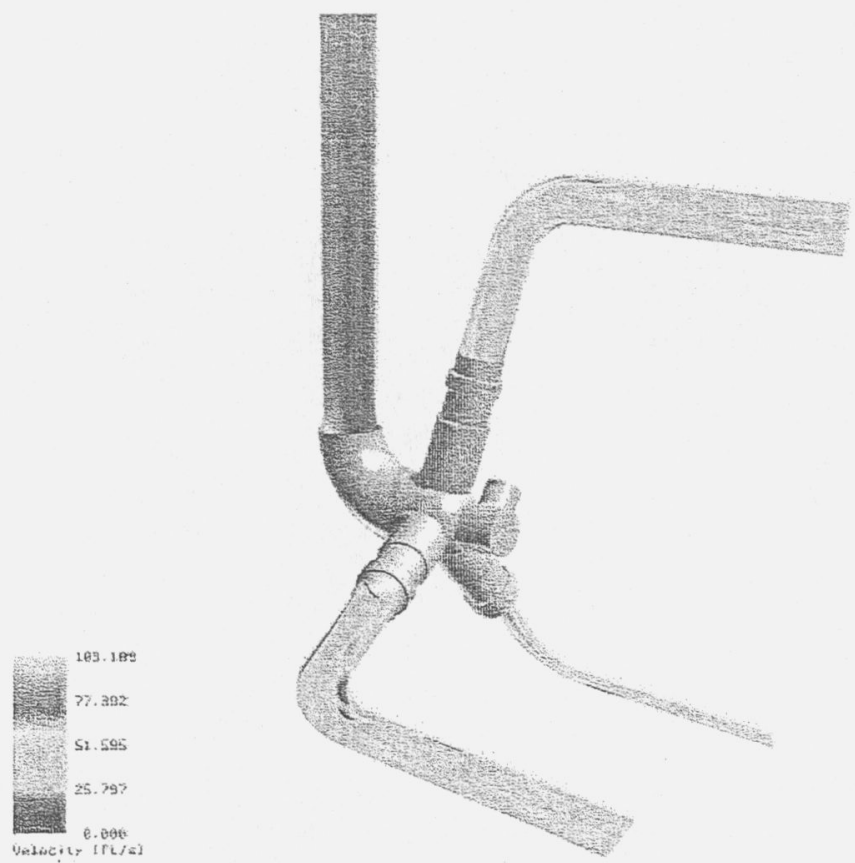

Figüre 16. Total velocity magnitude contours on the vertical planes of the orbiter manifold.
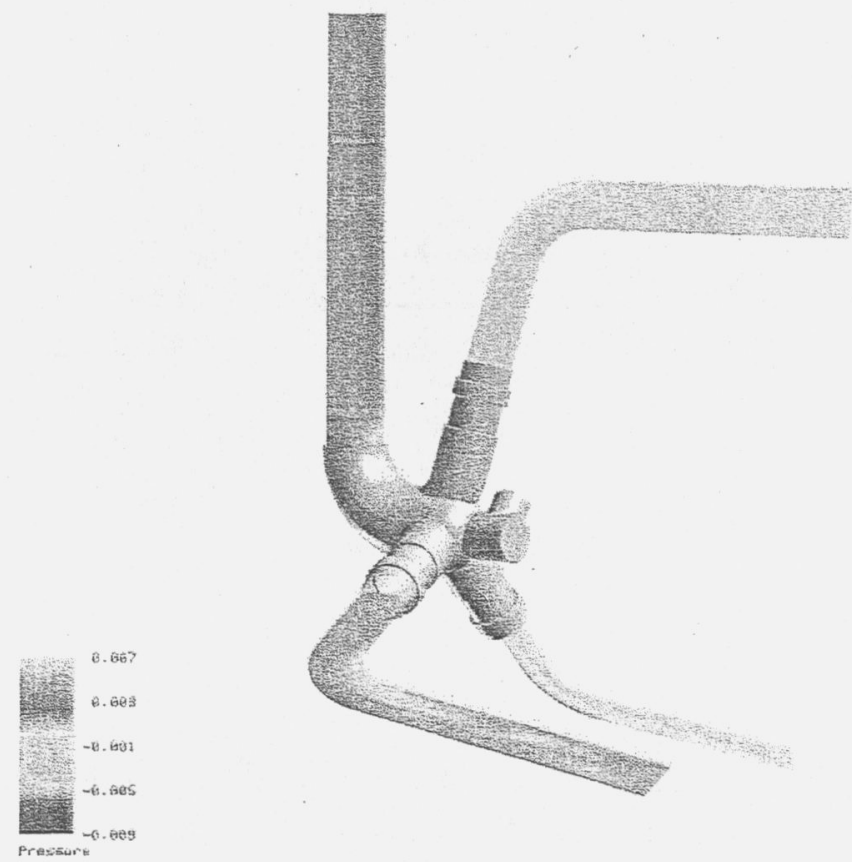

Figure 17. Pressure contours on the vertical planes of the orbiter manifold. 


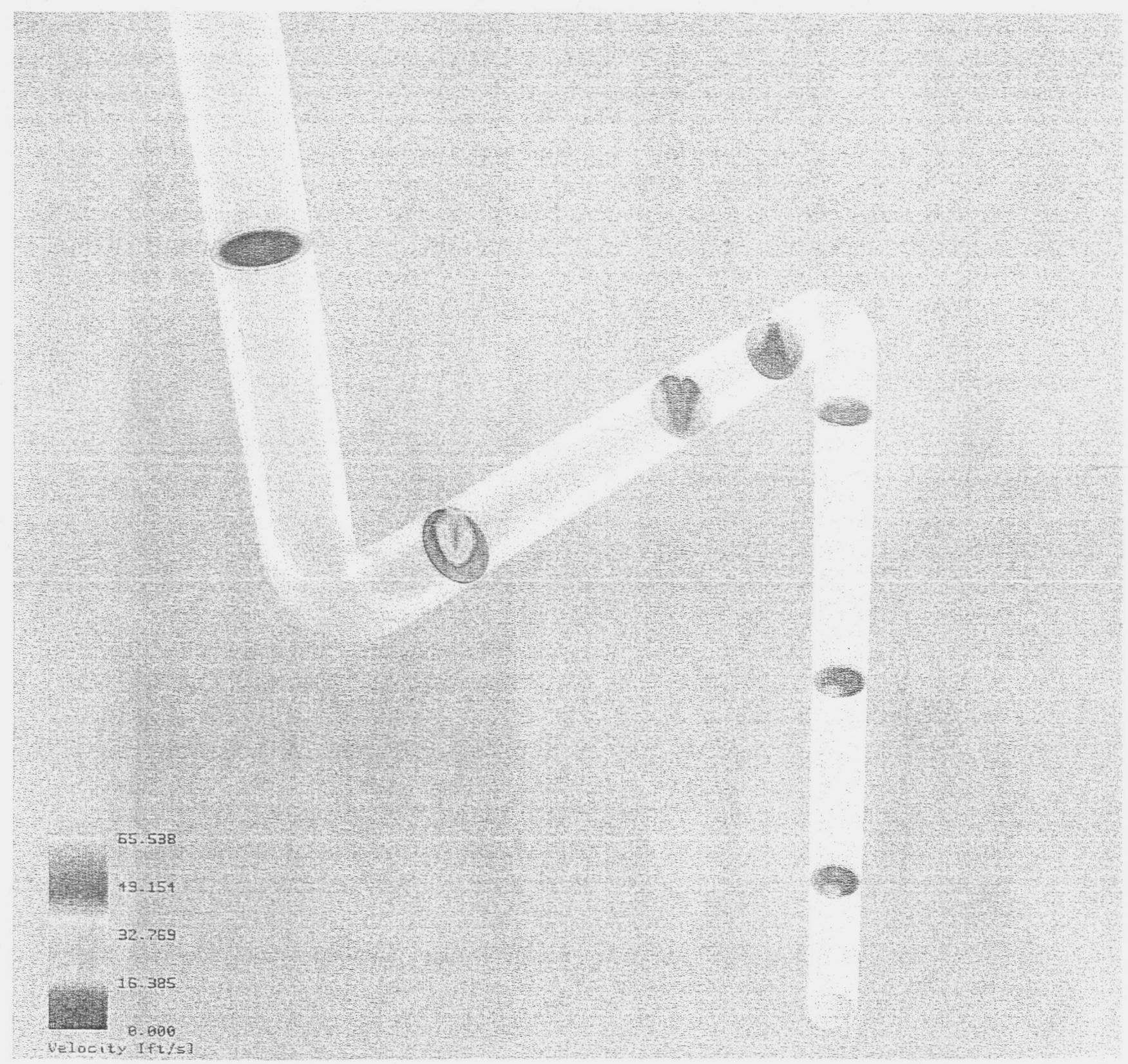

Figure 18. Total velocity magnitude contours at various cross-sections of the test article model

In figure 18 the velocity magnitude is displayed at various cross-sections of the test article. The flow direction is from the top of the figure to the bottom. The velocity profile is uniform before the first turn. Then it becomes non-uniform after the first turn, but it returns to a more uniform distribution toward the end of the pipe.

\section{Summary}

Inducer rotations are simulated in order to understand the root cause of the flowliner crack problem. CFD Fesults confirmed that there is a strong unsteady interaction between the backflow regions caused by the LPFTP inducer and secondary flow regions in the bellows cavity through the flowliner slots. It is observed that the swirl on the duct side of the downstream flowliner is stronger than on the duct side of the upstream flowliner. Due to this swirl, there are more significant unsteady flow interactions through 
the downstream slots than those observed in the upstream slots. A parametric study was performed to compare the flowfield in the flowliner area when one upstream slot and one corresponding downstream slot are enlarged. No significant differences were observed between the flowfield obtained from the enlarged slot configuration when compared with the original configuration. More cases must be analyzed with various enlarged slot configurations to generalize this observation. The flow through the test article and the flow through the orbiter fuel feedline manifold were simulated without the LPFTP. It was observed that incoming flow to the flowliner and inducer was more uniform in the test article then in the orbiter manifold. Additionally, each engine LPFTP in the orbiter receives significantly different velocity distributions. Because of these differences observed in the computed results it is not possible for the test article to represent the three different engine feedlines simultaneously.

\section{Acknowledgments}

The authors are grateful to Alex Tee for his help in CAD work, to Tim Sandstrom and to David Ellsworth for their help in the visualization work.

\section{References}

1. Chorin, A., J., "A Numerical Method for Solving Incompressible Viscous Flow Problems" Journal of Computational Physics, Vol. 2, pp. 12-26, 1967.

2. Kiris, C., and Kwak, D., 'Parallel Unsteady Turbopump Simulations for Reusable Launch vehicle,' Frontiers of Computational Fluid Dynamics 2002, Caughey, D.A. and Hafez, M., ed, World Scientific, 2002.

3. Kiris, C., Kwak, D., and Rogers, S., 'Incompressible Navier-Stokes Solvers in Primitive Variables and Their Applications to Steady and Unsteady Flow Simulations,' Numerical Simulations of Incompressible Flows, Hafez, M., ed, World Scientific, 2003.

4. Rogers, S. E., Kwak, D. and Kiris, C., "Numerical Solution of the Incompressible Navier-Stokes Equations for Steady and Time-Dependent Problems," AIAA Journal, Vol. 29, No. 4, pp. 603610, 1991.

5. Roe, P.L., "Approximate Riemann Solvers, Parameter Vectors, and Difference Schemes," J. of Comp. Phys., Vol. 43, pp. 357-372 1981.

6. MacCormack, R., W., "Current Status of Numerical Solutions of the Navier-Stokes Equations," AIAA Paper No. 85-0032, 1985. 